noma. J Clin Invest. 2014;124(3):1406-1417.

9. Ni M, Zhang Y, Lee AS. Beyond the endoplasmic reticulum: atypical GRP78 in cell viability, signalling and therapeutic targeting. Biochem J.
2011;434(2):181-188

10. Polier S, Samant RS, Clarke PA, Workman P, Prodromou C, Pearl LH. ATP -competitive inhibitors block protein kinase recruitment to the Hsp90-
Cdc37 system. Nat Chem Biol. 2013;9(5):307-312.

11. B'chir W, et al. The eIF $2 \alpha /$ ATF4 pathway is essential for stress-induced autophagy gene expression. Nucleic Acids Res. 2013;41(16):7683-7699.

\title{
Dynamin 2 the rescue for centronuclear myopathy
}

\author{
Alexis R. Demonbreun ${ }^{1}$ and Elizabeth M. McNally, ${ }^{1,2}$
}

${ }^{1}$ Department of Medicine and 2Department of Human Genetics, University of Chicago, Chicago, Illinois, USA.

\begin{abstract}
Centronuclear myopathy is a lethal muscle disease. The most severe form of the disease, $X$-linked centronuclear myopathy, is due to mutations in the gene encoding myotubularin (MTM1), while mutations in dynamin 2 (DNM2) and amphiphysin 2/BIN1 (AMPH2) cause milder forms of myopathy. MTM1 is a lipid phosphatase, and mutations that disrupt this activity cause severe muscle wasting. In this issue of the JCI, Cowling and colleagues report on their finding of increased DNM2 levels in human and mouse muscle with MTM1 mutations. Partial reduction of Dnm2 in mice harboring Mtm1 mutations remarkably rescued muscle wasting and lethality, and this effect was muscle specific. DNM2 regulates membrane trafficking through vesicular scission, and it is presumed that reducing this activity accounts for improved outcome in X-linked centronuclear myopathy.
\end{abstract}

\section{Defective membranes in centronuclear myopathies}

Centronuclear myopathy (CNM) is an inherited muscle-wasting disease with onset in infancy or childhood. Muscle biopsies from CNM patients characteristically show enlarged and centrally placed nuclei throughout the muscle, and clinically, CNM-associated mutations may result in weakened respiratory musculature, leading to early death without supportive care. Three main forms of CNM are attributable to mutations in myotubularin (MTM1), amphiphysin 2 (AMPH2), and dynamin 2 (DNM2), which all encode membraneassociated proteins that potentially act in muscle at the transverse tubules (T-tubules), membrane invaginations specialized for calcium handling (Figure 1 and ref. 1). The most common and severe form of CNM is X-linked CNM (XLCNM), referred to as myotubular myopathy. XLCNM is clinically evident at birth, with patients presenting with hypotonia and respiratory compromise. Skeletal muscles biopsies from XLCNM patients show abnormal T-tubules, including defective triads, struc-

Conflict of interest: The authors have declared that no conflict of interest exists.

Citation for this article: J Clin Invest. 2014; 124(3):976-978. doi:10.1172/JCI74434. tures critical for excitation-contraction coupling. XLCNM is the result of mutations in MTM1, which encodes a ubiquitously expressed phosphoinositide 3-phosphatase that functions to dephosphorylate PI3P and phosphatidylinositol-3,4-bisphosphate, two phospholipids essential for membrane sorting and signaling (2). MTM1 is required for normal endocytosis, receptor degradation, and early endosome maturation (1).

Mice harboring a deletion of Mtm1 (Mtm1-/y mice) recapitulate human disease and display many characteristic XLCNMassociated features, including muscle weakness, centralized nuclei, and muscle atrophy (3). Mtm $1^{-/ y}$ mice exhibit muscle atrophy, become weak at 3 to 4 weeks of age, and have a greatly reduced life expectancy of only 6 to 12 weeks (3). The role of MTM1 in human muscle has also been effectively modeled in zebrafish using morpholinos to reduce $m t m 1$ expression (MTM MO) (4). MTM $\mathrm{MO}$ fish, like XLCNM patients and $\mathrm{Mtm}^{-/} \mathrm{H}^{-1}$ mice, develop weakness and muscle atrophy. MTM MO fish display accumulation of PI3P, especially surrounding muscle nuclei, and T-tubule defects. The phenotypes observed in MTM MO fish provide further support that MTM1 is critical for the normal biogenesis and maintenance of membrane structures within muscle (4). Because of its broad role as a lipid phosphatase, the indispensable targets of MTM1's enzymatic action are not fully known.

DNM2 is a ubiquitously expressed GTPase that has been implicated in multiple cellular functions, including endocytosis, membrane scission, and cytoskeletal remodeling (5). Dynamins assemble as rings around membrane tubules where they are thought to actively "pinch off" membranes (5). Complete loss of Dnm2 results in embryonic lethality in mice (6). In humans, CNM is caused by dominant DNM2 mutations (7), and overexpression of a CNM-linked DNM2 mutation (R465W) in mouse muscle results in myopathic features, including centralized nuclei, muscle atrophy, and deformed T-tubules (8). Interestingly, overexpression of normal DNM2 in mouse models also produces some of these same features, consistent with the model that increased DNM2 activity contributes to CNM (8). Increased DNM2 activity may lead to excessive membrane scission and pruning, giving the appearance of excessive membrane accumulation around nuclei or at T-tubules.

\section{Genetic reduction of DNM2 in a CNM model}

In this issue of the JCI, Cowling and colleagues described elevated protein levels of DNM2 in $M t m 1^{-/ y}$ animals and in muscle biopsies from human XLCNM patients (9). To target the increased levels of DNM2 found in $M t m 1^{-/ y}$ animals, mice heterozygous for Dnm2 were crossed with $M t m 1^{-1 y}$ mice. The in vivo reduction of Dnm2 corrected some histological abnormalities in muscle, but dramatically extended life expectancy from 6 to 12 weeks to beyond one year. To demonstrate that this effect was muscle intrinsic, Cowling and colleagues generated $M t m 1^{-/ y}$ mice in which $D n m 2$ was specifically reduced in skeletal muscle. Reduction of Dnm2 in skeletal muscle alone after disease onset was sufficient to reduce pathology and 


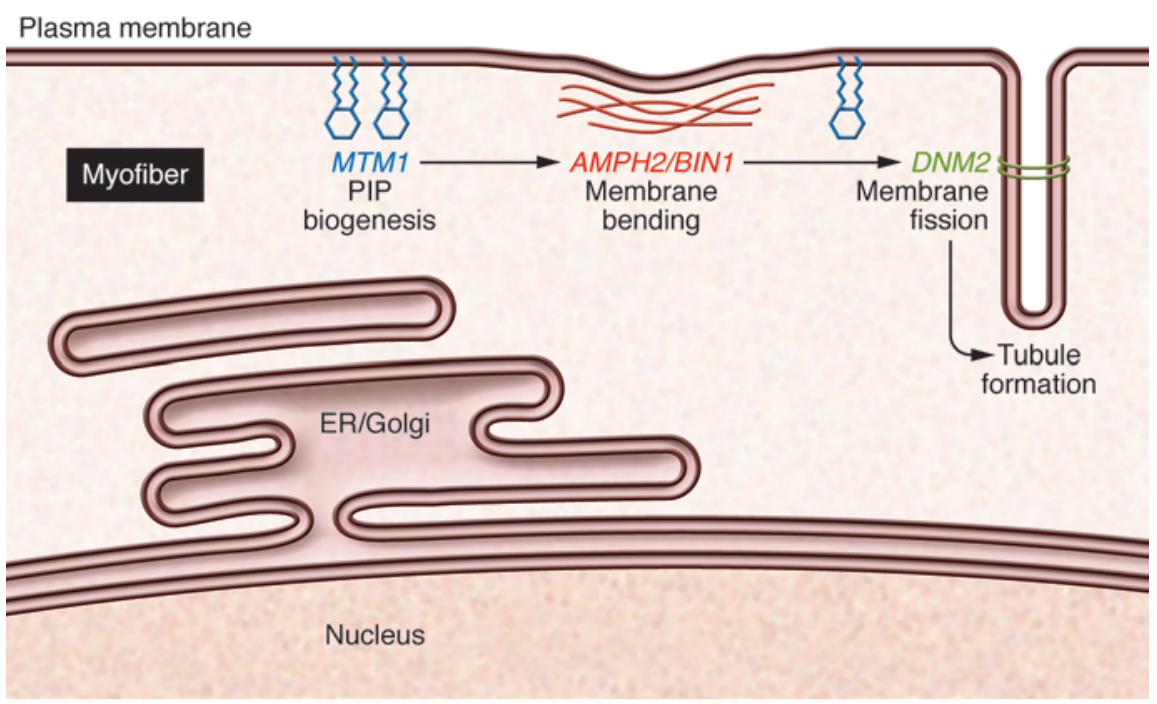

extend the life span in $M t m 1^{-/ y}$ mice; however, skeletal muscle-specific Dnm2 deletion did not extend life span in $M t m 1^{-/ y}$ animals to the same extent as heterozygous wholebody Dnm2 reduction, suggesting that aspects of the phenotype arise from muscle extrinsic effects or the need to reduce Dnm2 earlier in muscle development.

Based on their data, Cowling et al. propose that DNM2 and MTM1 function in an overlapping pathway, but there are no reports of a direct interaction between these two proteins. DNM2 is enriched at the $\mathrm{Z}$ band of muscle, adjacent to but not directly overlapping with T-tubules, and DNM2 is also seen in the membranes surrounding myonuclei $(8,10)$. Many DNM2 mutations fall near the pleckstrin homology domain, which may insert directly into membranes to participate in membrane fission. These observations suggest a broader complex may be critical for the restricted action of MTM1 and DNM2 on membrane biogenesis and function. The large size of DNM2 may require alternative approaches to provide adequate resolution to fully define its associated membranes in muscle.

AMPH2, also known as BIN1 (bridging integrator protein), is a Bin-AmphiphysinRvs (BAR) domain-containing protein, known to sense membrane curvature and to bend membranes (1). Membrane bending is a key aspect of forming intracellular tubules in all cell types and also in muscle. Recent work has shown that BIN1 binds phosphatidylinositol-4,5-bisphosphate at the T-tubule and is capable of binding both DNM2 and MTM1 $(11,12)$. The binding of MTM1 to BIN1 enhances tubule formation in vitro, and mutations in MTM1 that cause $\mathrm{CNM}$ result in reduced tubule formation in vitro. MTM1-deficient muscle lacks normal BIN1 localization at the T-tubule (12); therefore, MTM1 loss may result in defective membrane biogenesis through upregulation and altered localization of phosphoinositides. The increase in DNM2 observed in XLCNM patients may arise as a consequence of altered lipid signaling.

Currently, there are no drug therapies for CNM patients, and care relies on respiratory support and management. To date, most therapeutic approaches have focused on MTM1 replacement. For example, local restoration of MTM1 with viral delivery improved both the structural and functional deficits of Mtm1 deficiency in mice (13). Alternatively, direct enzyme replacement therapy of MTM1 in mice markedly improved muscle strength after only two weeks of treatment (14). These preclinical approaches for MTM1 replacement are promising, but have yet to be tested in a clinical setting.

The improvement in strength and pathology following Dnm2 reduction in $\mathrm{Mtm}^{-/ y}$ mice suggests that targeting DNM2 is an alternative and potentially complementary strategy for XLCNM therapy. Importantly, the decrease in XLCNM-associated phenotypes observed following DNM2 reduction after disease onset is essential for any XLCNM treatment, given that most patients present with disease at birth. The in vivo overexpression and loss-of-function studies in mice suggest a wide therapeutic window in which to manipulate DNM2 levels safely in clinic for XLCNM treatment. Molecular strategies to inhibit DNM2 could also be applied to other forms of CNM. For example, the cell-permeable molecule dynasore and the

\section{Figure 1}

Genes implicated in CNM. MTM1 encodes a membrane-bound lipid phosphatase (blue). Loss of function MTM1 mutations lead to severe, early onset myopathy characterized by myofibers with centrally placed nuclei. Dominant mutations in DNM2, encoding dynamin 2 (green), also lead to CNM. In this issue, Cowling and colleagues demonstrate that reducing DNM2 dramatically improves longevity in a mouse model of MTM1-mediated myopathy. Mutations in AMPH2/BIN1 (red) also cause CNM. These proteins are involved in phosphoinositide (PIP) biogenesis, membrane bending, and membrane fission. Mutations in these genes affect membranes throughout the muscle, including those that surround nuclei and those in the T-tubule.

serotonin reuptake inhibitor sertraline both act as reversible noncompetitive inhibitors that block the GTPase activity of DNM2, resulting in diminished endocytosis $(5,15)$. Unfortunately, these molecules lack specificity and would interact with other dynamins, including dynamin 1 and the mitochondrial dynamin DRP1 $(5,15)$. Whether other dynamins are increased and/or contribute to pathology in XLCNM is not known. Downregulating multiple dynamins within and outside of muscle could increase the risk of unwanted off-target effects. Mice with deletions of microRNA 133a-1 (Mir133a-1) and Mir133a-2 develop a CNM that is mediated by upregulation of Dnm2 (16). Because $D n m 2$ is a direct target of Mir133a, it is possible that this microRNA could be used to reduce DNM2 levels in CNM patients.

\section{Can reduction of DNM2 be extended as a therapy in other myopathies?}

The rescue of XLCNM through the genetic reduction of DNM2 is a viable approach for this rare form of muscle disease; however, many genetically diverse forms of inherited myopathies share aspects of the phenotypes seen in CNM. For example, mutations in dysferlin (DYSF) and caveolin 3 (CAV3) also result in some histological abnormalities shared with CNM, including T-tubule defects and muscle weakness $(17,18)$. Similarly to MTM1, DYSF and CAV3 also regulate endocytosis and colocalize with BIN1 at the T-tubule (19-21). Herein, Cowling and colleagues showed abnormal internalization of CAV3 in $\mathrm{Mtm}^{-/ y}$ muscle, which was rescued by $D n m 2$ reduction. Interestingly, both overexpression of normal DNM2 in mice and DNM2 loss-of- 
function mutations in mice and humans results in abnormal DYSF internalization $(10,16)$. Whether mislocalization of CAV3 and DYSF within the myofiber contributes to CNM disease progression remains to be determined. These data suggest that a common pathway links MTM1, DNM2, BIN1, DYSF, and CAV3 in the biogenesis and maintenance of muscle, specifically at the T-tubule. If DNM2 levels are found to be upregulated in these other forms of myopathy, then targeting DNM2 becomes a common therapeutic strategy for a wider range of muscle disease.

\section{Acknowledgments}

This work was supported by NIH grants NS047726, AR053646, and NS072027.

Address correspondence to: Elizabeth M. McNally, University of Chicago, 5841 S. Maryland, MC6088, Chicago, Illinois 60637, USA. Phone: 773.702.2684; Fax: 773.702.2681; E-mail: emcnally@ uchicago.edu.

1. Dowling JJ, Gibbs EM, Feldman EL. Membrane traffic and muscle: lessons from human disease.
Traffic. 2008;9(7):1035-1043.

2. De Matteis MA, Godi A. PI-loting membrane traffic. Nat Cell Biol. 2004;6(6):487-492.

3. Buj-Bello A, et al. The lipid phosphatase myotubularin is essential for skeletal muscle maintenance but not for myogenesis in mice. Proc Natl Acad Sci U S A. 2002;99(23):15060-15065.

4. Dowling JJ, et al. Loss of myotubularin function results in T-tubule disorganization in zebrafish and human myotubular myopathy. PLoS Genet. 2009;5(2):e1000372.

5. Macia E, Ehrlich M, Massol R, Boucrot E, Brunner C, Kirchhausen T. Dynasore, a cell-permeable inhibitor of dynamin. Dev Cell. 2006;10(6):839-850.

6. Ferguson SM, et al. Coordinated actions of actin and BAR proteins upstream of dynamin at endocytic clathrin-coated pits. Dev Cell. 2009;17(6):811-822.

7. Bitoun $\mathrm{M}$, et al. Mutations in dynamin 2 cause dominant centronuclear myopathy. Nat Genet. 2005;37(11):1207-1209.

8. Cowling BS, et al. Increased expression of wild-type or a centronuclear myopathy mutant of dynamin 2 in skeletal muscle of adult mice leads to structural defects and muscle weakness. Am J Pathol. 2011; 178(5):2224-2235.

9. Cowling BS, et al. Reducing dynamin 2 expression rescues $\mathrm{X}$-linked centronuclear myopathy. J Clin Invest. 2014;124(3):1350-1363.

10. Durieux AC, et al. A centronuclear myopathydynamin 2 mutation impairs skeletal muscle structure and function in mice. Hum Mol Genet. 2010; 19(24):4820-4836.

11. Kojima C, et al. Regulation of Bin $1 \mathrm{SH} 3$ domain binding by phosphoinositides. EMBO J. 2004; 23(22):4413-4422.

12. Royer B, Hnia K, Gavriilidis C, Tronchere H, Tosch $\mathrm{V}$, Laporte J. The myotubularin-amphiphysin 2 complex in membrane tubulation and centronuclear myopathies. EMBO Rep. 2013;14(10):907-915.

13. Buj-Bello A, et al. AAV-mediated intramuscular delivery of myotubularin corrects the myotubular myopathy phenotype in targeted murine muscle and suggests a function in plasma membrane homeostasis. Hum Mol Genet. 2008;17(14):2132-2143.

14. Lawlor MW, et al. Enzyme replacement therapy rescues weakness and improves muscle pathology in mice with X-linked myotubular myopathy. Hum Mol Genet. 2013;22(8):1525-1538

15. Takahashi K, Miyoshi H, Otomo M, Osada K, Yamaguchi N, Nakashima H. Suppression of dynamin GTPase activity by sertraline leads to inhibition of dynamin-dependent endocytosis. Biochem Biophys Res Commun. 2010;391(1):382-387.

16. Liu N, et al. Mice lacking microRNA 133a develop dynamin 2-dependent centronuclear myopathy. J Clin Invest. 2011;121(8):3258-3268.

17. Galbiati F, et al. Caveolin-3 null mice show a loss of caveolae, changes in the microdomain distribution of the dystrophin-glycoprotein complex, and t-tubule abnormalities. J Biol Chem. 2001; 276(24):21425-21433

18. Klinge L, et al. Dysferlin associates with the developing T-tubule system in rodent and human skeletal muscle. Muscle Nerve. 2010;41(2):166-173.

19. Demonbreun AR, Fahrenbach JP, Deveaux K, Earley JU, Pytel P, McNally EM. Impaired muscle growth and response to insulin-like growth factor 1 in dysferlin-mediated muscular dystrophy. Hum Mol Genet. 2011;20(4):779-789.

20. Lee E, et al. Amphiphysin 2 (Bin1) and T-tubule biogenesis in muscle. Science. 2002;297(5584):1193-1196.

21. Kerr JP, et al. Dysferlin stabilizes stress-induced $\mathrm{Ca} 2+$ signaling in the transverse tubule membrane. Proc Natl Acad Sci U S A. 2013;110(51):20831-20836.

\title{
PPAR $y$ in emphysema: blunts the damage and triggers repair?
}

\author{
Neil J. Kelly and Steven D. Shapiro
}

Division of Pulmonary, Allergy, and Critical Care, Department of Medicine, UPMC and University of Pittsburgh, Pittsburgh, Pennsylvania, USA.

Cigarette smoke is the most common cause of pulmonary emphysema, which results in an irreversible loss of lung structure and function. Th1 and Th17 immune responses have been implicated in emphysema pathogenesis; however, the drivers of emphysema-associated immune dysfunction are not fully understood. In this issue of the JCI, Shan and colleagues found that peroxisome proliferator-activated receptor $\gamma(\operatorname{PPAR} \gamma)$ is downregulated in APCs isolated from the lungs of emphysematous chronic smokers and mice exposed to cigarette smoke. Furthermore, treatment with a PPAR $\gamma$ agonist prevented emphysema development and appeared to reduce emphysemaassociated lung volume expansion in mice exposed to cigarette smoke. Further work will need to be done to evaluate the potential of PPAR $\gamma$ agonists to restore lung capacity in emphysematous patients.

Pulmonary emphysema is a major component of chronic obstructive pulmonary dis-

Conflict of interest: The authors have declared that no conflict of interest exists.

Citation for this article: J Clin Invest. 2014; 124(3):978-980. doi:10.1172/JCI74417. ease (COPD) and involves the loss of alveolar units distal to the terminal bronchioles. Even though COPD holds an unenviable position as the world's fourth-leading cause of death, current medical interventions have little to offer beyond symptomatic relief. Meanwhile, the prevalence of
COPD is expected to continue to rise as low- and middle-income countries join in the developed world's tobacco addiction. If we are to avoid this grim projection, we must expand our knowledge of the basic mechanisms behind lung injury and repair before translating these findings into novel therapeutic treatments.

\section{Pulmonary emphysema results from an imbalance between elastases and anti-elastases}

It has been more than 50 years since Laurell and Eriksson first identified a deficiency in $\alpha-1$ antitrypsin, the major inhibitor of neutrophil elastase (ELANE), as the culprit behind hereditary pulmonary emphysema (1). Since the involvement of ELANE in pulmonary emphysema was first reported, our understanding of the disease's 